Supporting information of

\title{
Revealing the Effects of the Non-Solvent on the Ligand Shell of Nanoparticles and Their Crystallization
}

\author{
Byeongdu Lee, ${ }^{{ }^{*}}$ Kenneth Littrell, ${ }^{\bar{\top}}$ Yuchen Sha ${ }^{\ddagger \perp}$ and Elena V. Shevchenko ${ }^{\ddagger *}$ \\ ${ }^{*}$ Advanced Photon Source, Argonne National Laboratory, Argonne, Illinois 60439 USA; \\ ${ }^{\bar{\top}}$ Neutron Sciences Directorate, Oak Ridge National Laboratory, Oak Ridge, TN 37831; \\ ${ }^{\perp}$ Institute of Advanced Studies (IAS), College of Chemistry and Molecular Sciences, Wuhan University, \\ Wuhan 430072, Hubei, P. R. China; \\ ${ }^{\ddagger}$ Center for Nanoscale Materials, Argonne National Laboratory, Argonne, Illinois 60439 USA;
}

Corresponding authors: Byeongdu Lee (blee@anl.gov) and Elena V. Shevchenko (eshevchenko@anl.gov).

\section{Table of Contents}

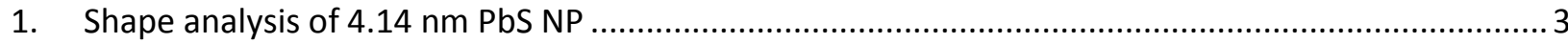

2. Mathematical relation between the sphere radius and truncated octahedron edge length

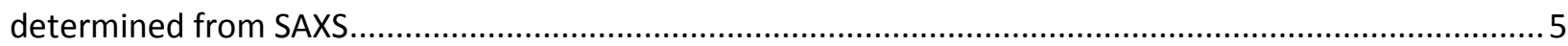

a. A sphere and a truncated octahedron with the same $\mathrm{Rg}$........................................................ 5

b. Radius of the circumscribed sphere of a truncated octahedron ................................................... 5

3. Math equations for interparticle spacing calculation .................................................................. 6

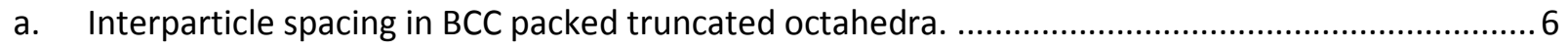

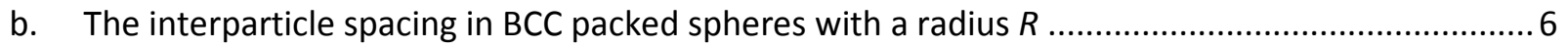

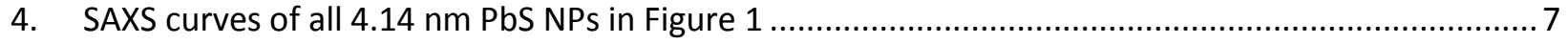

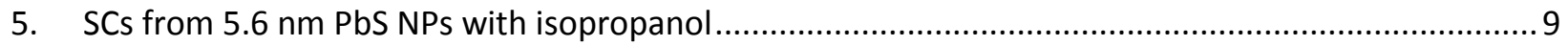

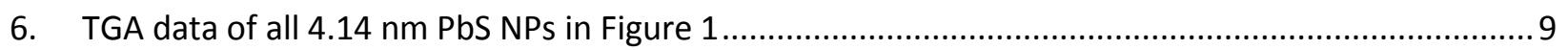

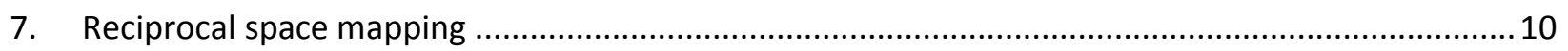

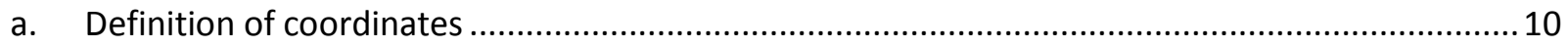

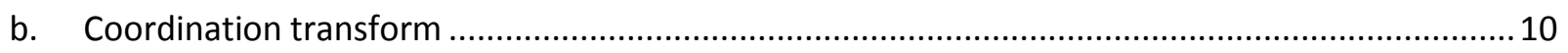

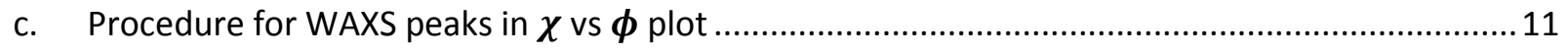

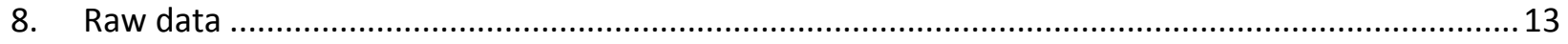




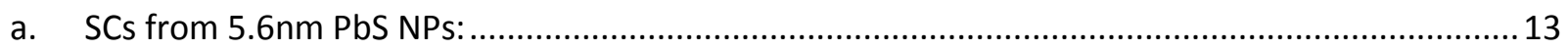

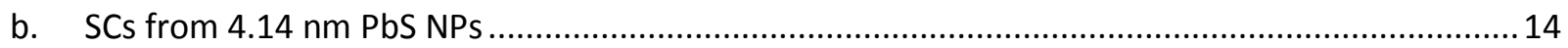

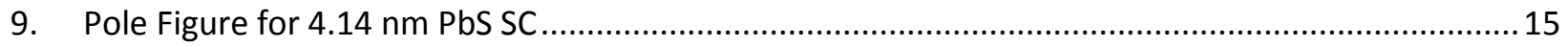

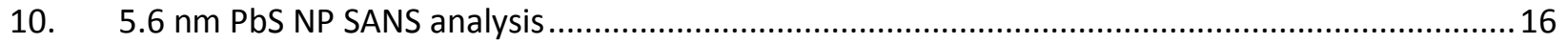

11. Comments on solvent/non-solvent purification technique:.................................................... 18

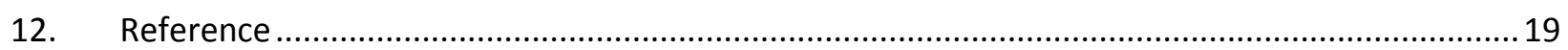




\section{Shape analysis of $4.14 \mathrm{~nm}$ PbS NP}

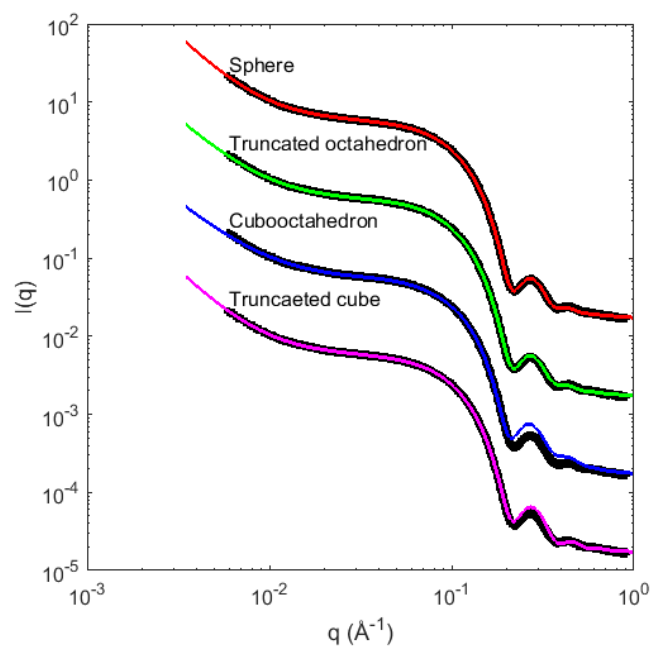

Figure S1. Best fit of SAXS data from a batch of $4.14 \mathrm{~nm}$ PbS NP in toluene with various polyhedra models.

Table S1. Results of best fit shown in Figure S1.

\begin{tabular}{|c|c|c|c|c|c|c|c|}
\hline Shape & Model & $\begin{array}{c}\text { Radius (R) } \\
\text { or Edge } \\
\text { length (L) }\end{array}$ & $\begin{array}{c}\text { Distributi } \\
\text { on width }\end{array}$ & $\begin{array}{c}\text { Surface } \\
\text { Area (S) }\end{array}$ & $\begin{array}{c}\text { Volume } \\
\text { (V) }\end{array}$ & $\mathrm{S} / \mathrm{V}^{\mathrm{a}}$ & $\mathrm{Sp}^{\mathrm{b}}$ \\
\hline Sphere & $20.38 \AA$ & $1.96 \AA$ & $4 \pi R^{2}$ & $\frac{4 \pi}{3} R^{3}$ & 0.1472 & 1 \\
\hline $\begin{array}{c}\text { Truncated } \\
\text { octahedro } \\
\mathrm{n}\end{array}$ & $14.59 \AA$ & $1.34 \AA$ & $(6+12 \sqrt{3}) L^{2}$ & $8 \sqrt{2} L^{3}$ & 0.1623 & 0.9099 \\
\hline $\begin{array}{c}\text { Cuboocta } \\
\text { hedron }\end{array}$ & $24.48 \AA$ & $2.49 \AA$ & $(6+2 \sqrt{3}) L^{2}$ & $\frac{5}{3} \sqrt{2} L^{3}$ & 0.1640 & 0.9050 \\
\hline $\begin{array}{c}\text { Truncated } \\
\text { cube }\end{array}$ & $13.74 \AA$ & $0.95 \AA$ & $\begin{array}{c}2(6+6 \sqrt{2} \\
+\sqrt{3}) L^{2}\end{array}$ & $\frac{(21+14 \sqrt{2})}{3} L^{3}$ & 0.1736 & 0.8495 \\
\hline
\end{tabular}

${ }^{a} \mathrm{~S}$ (Surface area) and $\mathrm{V}$ (volume) are not mean values for determined size distributions, and the ratios do not consider particle size distributions.

a Sphericity $=\pi^{\frac{1}{3}}(6 V)^{\frac{2}{3}} / S$ 
Table S2. SAXS analysis of particles used for in the work

\begin{tabular}{|c|c|c|c|c|c|c|}
\hline \multirow{2}{*}{$\begin{array}{c}\text { Sample } \\
\text { Nomnclature }\end{array}$} & \multicolumn{2}{|c|}{ Sphere } & \multicolumn{2}{c|}{ Cuboctahedron } & \multicolumn{2}{c|}{ Truncated octahedron } \\
\cline { 2 - 7 } & Radius $(\AA)$ & $\begin{array}{c}\text { Distribution } \\
\text { width }(\AA)\end{array}$ & $\begin{array}{c}\text { Edge } \\
\text { length } \\
(\AA)\end{array}$ & $\begin{array}{c}\text { Distribution } \\
\text { width }(\AA)\end{array}$ & $\begin{array}{c}\text { Edge } \\
\text { length }(\AA)\end{array}$ & $\begin{array}{c}\text { Distribution } \\
\text { width }(\AA)\end{array}$ \\
\hline $4.14 \mathrm{~nm} \mathrm{NP}$ & 20.7 & 1.93 & $24.2 \pm 1$ & $2.0 \pm 0.5$ & 14.8 & 1.21 \\
\hline $5.6 \mathrm{~nm} \mathrm{NP}$ & 27.16 & 5.52 & $28.5 \pm 1$ & $6.6 \pm 0.5$ & 19.1 & 3.94 \\
\hline
\end{tabular}

Details of fitting formula and procedures are found in the literature. ${ }^{1}$ 


\section{Mathematical relation between the sphere radius and truncated octahedron edge length determined from SAXS}

Radius of gyration $(\mathrm{Rg})$ of a truncated octahedron for $8 \sqrt{2} L_{\text {to }}^{3}=1:^{2}$

$$
R_{g}=0.48542
$$

Rg of a sphere:

$$
R_{g}=\sqrt{\frac{3}{5}} R
$$

a. A sphere and a truncated octahedron with the same Rg

When both a sphere and truncated octahedron can fit a SAXS curve, the mathematical ratio of the sphere radius and the edgelength of the truncated octahedron is

$$
R=0.48542 \cdot \frac{5^{\frac{1}{2}} 8^{\frac{1}{3}} 2^{\frac{1}{6}}}{3^{\frac{1}{2}}} L_{t o}
$$

For a sphere with diameter $4.14 \mathrm{~nm}, L_{t o}=1.4714$. For this tuncated ocahedron, face-face distance between parallel 111 planes is $3.604 \mathrm{~nm}$. The face-face distance between 001 planes is $4.162 \mathrm{~nm}$.

For a sphere with diameter $5.6 \mathrm{~nm}, L_{t o}=1.9903$. For this tuncated ocahedron, face-face distance between parallel 111 planes is $4.875 \mathrm{~nm}$. The face-face distance between 001 planes is $5.629 \mathrm{~nm}$.

b. Radius of the circumscribed sphere of a truncated octahedron

When the edgelength of a truncated octahedron is $L_{t o}$, radius of the circumscribed sphere of the polyhedron is

$$
R=\frac{\sqrt{10}}{2} L_{t o}
$$




\section{Math equations for interparticle spacing calculation}

a. Interparticle spacing in BCC packed truncated octahedra.

When the lattie constant of a $\mathrm{BCC}$ is $a_{B C C}$, Center-to-center distance between the nearest particles of $\mathrm{BCC}$ is $\frac{a_{B C C} \sqrt{3}}{2}$.

When the edge length of a truncated octahedron is $L_{t o},(111)$ to (111) plane distance of an octahedron is $2.4495 L_{t o}$. Then, the interparticle spacing between two nearest NPs packed in an ideal configuration (Figure $2 \mathrm{~h}$ ) is

$$
\mathrm{d}_{\text {interparticle spacing }}=\frac{a_{B C C} \sqrt{3}}{2}-2.4495 L_{t o}
$$

b. The interparticle spacing in BCC packed spheres with a radius $R$

$$
\mathrm{d}_{\text {interparticle spacing }}=\frac{a_{B C C} \sqrt{3}}{2}-2.0 R
$$




\section{SAXS curves of all $4.14 \mathrm{~nm}$ PbS NPs in Figure 1}

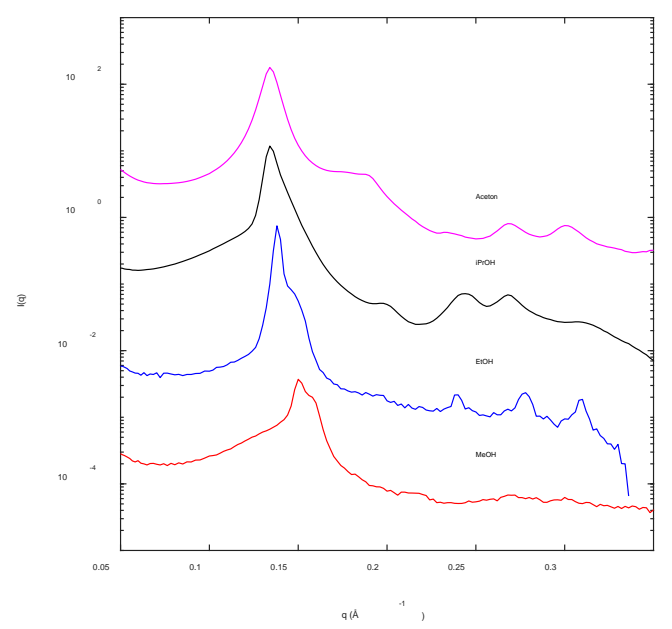

Figure S2. SAXS curves of $4.14 \mathrm{~nm}$ PbS SC with various non-solvents.

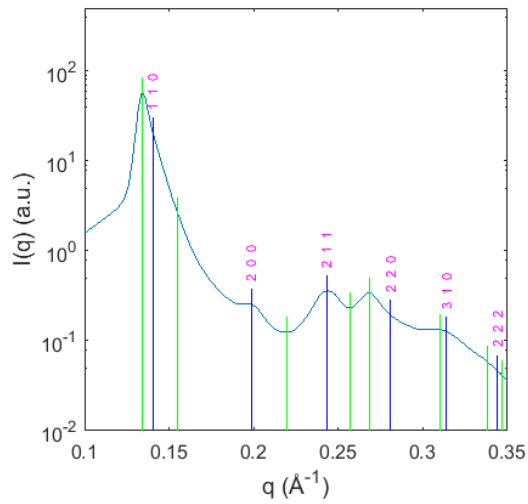

Figure S3. $4.14 \mathrm{~nm}$ PbS NP with isopropanol and indexing of $\mathrm{fcc}$ and bcc lattices. Blue lines) bcc indexing, $\mathrm{a}_{\mathrm{SL}}=63.29 \AA$. Green line) $\mathrm{fcc}$ indexing with $\mathrm{a}_{\mathrm{SL}}=81.04 \AA$. Interparticle spacing $=15.21 \AA$ and $17.70 \AA$ for bcc and fcc, respectively. 

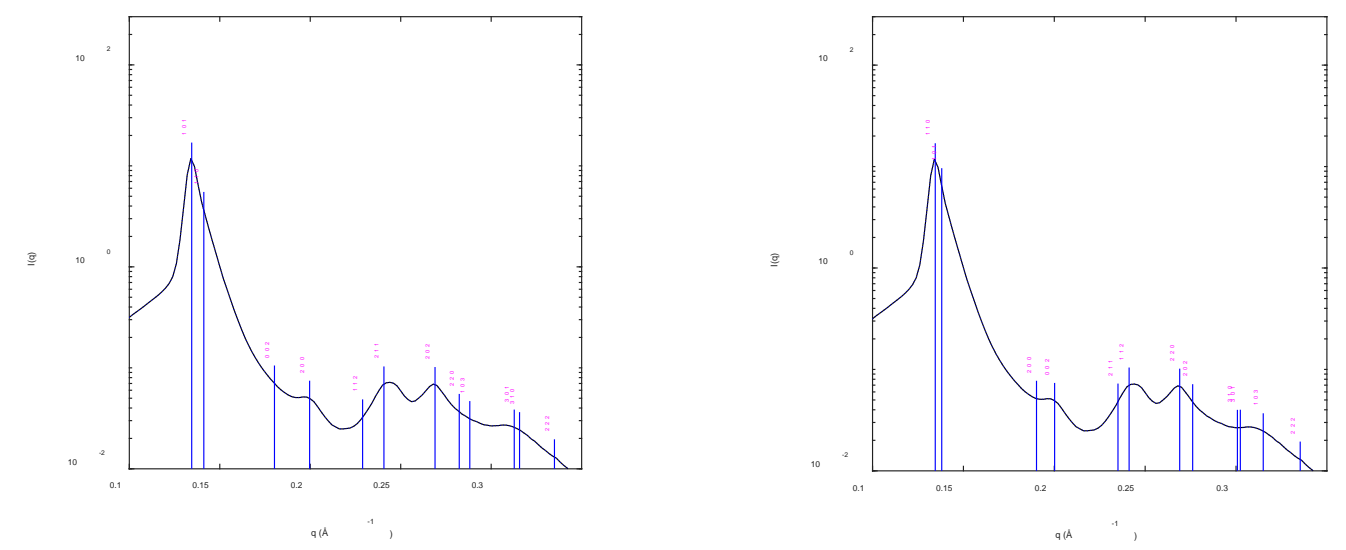

Figure S4. $4.14 \mathrm{~nm}$ PbS NP with isopropanol and bct indexing. left) $\mathrm{a}_{\mathrm{SL}}=62.9 \AA$ and $\mathrm{C}_{S \mathrm{~L}}=69.7 \AA$ and right) $\mathrm{a}_{\mathrm{SL}}=66.1$ and $\mathrm{CSL}_{\mathrm{SL}}=62.8 \AA$.
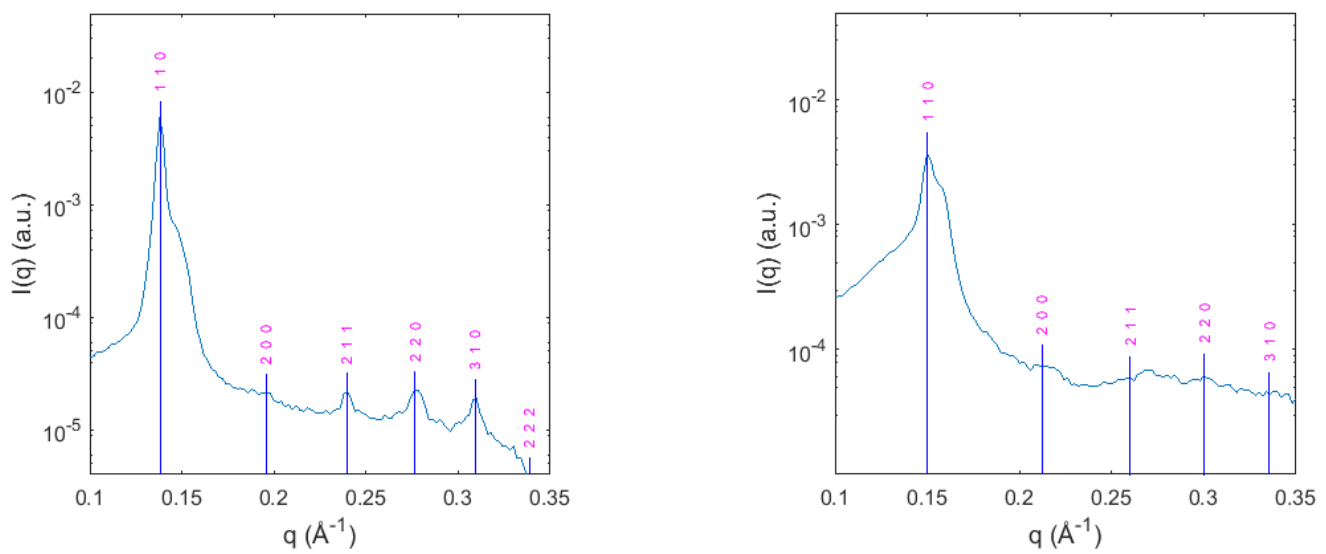

Figure S5. $4.14 \mathrm{~nm}$ PbS NP with ethanol (left) and methanol (right). Indexing with bcc symmetry. Left) ast $=64.26 \AA$. Interparticle spacing $=16.05 \AA$. right) $\mathrm{a}_{\mathrm{SL}}=59.22 \AA$. Interparticle spacing $=11.69 \AA$. 


\section{SCs from $5.6 \mathrm{~nm}$ PbS NPs with isopropanol}
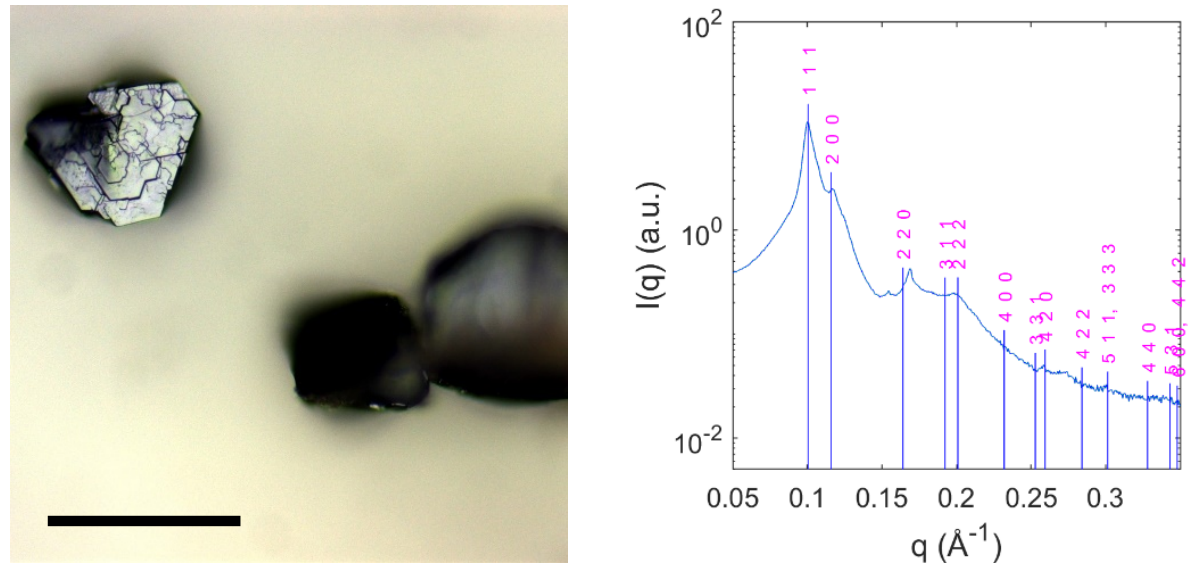

Figure S6. SCs from $5.6 \mathrm{~nm}$ PbS NPs with isopropanol. Left) optical image with 100um scale bar. Right):

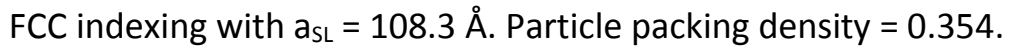

6. TGA data of all $4.14 \mathrm{~nm}$ PbS NPs in Figure 1

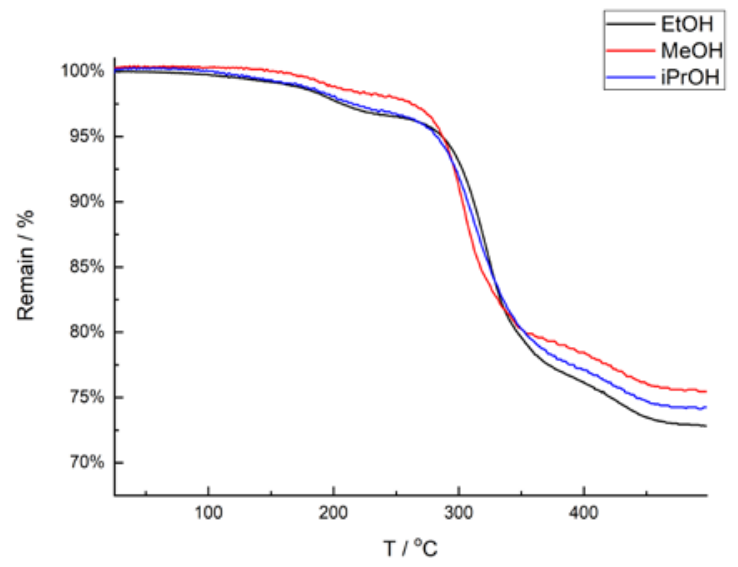

Figure S7. TGA data obtained on dried SCs prepared by destabilization of $4.14 \mathrm{~nm}$ PbS NP with methanol, ethanol and isopropanol. 


\section{Reciprocal space mapping}

a. Definition of coordinates

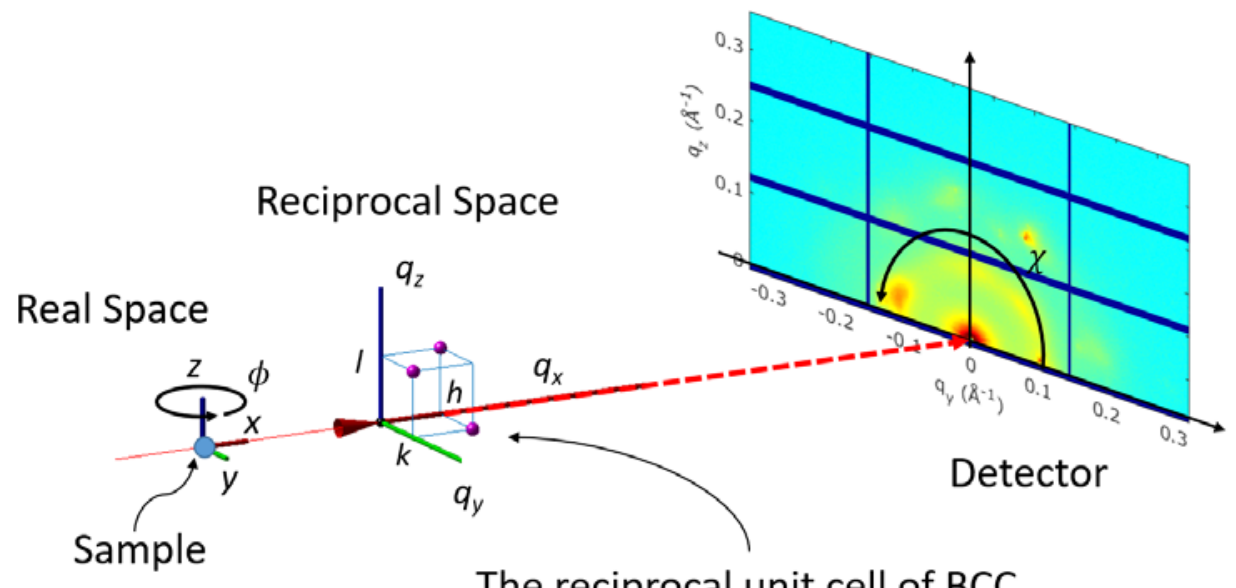

Figure S8. Coordinates and notations for angles for x-ray measurement

b. Coordination transform

Here we provide the relation between a pixel on a detector to a position in the reciprocal space, $q$. The scattering vector is

$$
\boldsymbol{q}=\left(q_{x}, q_{y}, q_{z}\right)
$$

And

$$
\begin{gathered}
q_{x}=k_{0}(\cos \alpha \cos (\theta+\phi)-\cos \phi) \\
q_{y}=k_{0}(\cos \alpha \sin (\theta+\phi)-\sin \phi) \\
q_{z}=k_{0} \sin \alpha
\end{gathered}
$$

, where $k_{0}=2 \pi / \lambda$ and $\lambda$ is the wavelength of $x$-ray. The length of the scattering vector is $q=|\boldsymbol{q}|=$ $\sqrt{q_{x}^{2}+q_{y}^{2}+q_{z}^{2}}$

If a detector is perpendicular to the x-ray beam,

and

$$
\alpha=\tan ^{-1} \frac{Z p}{\sqrt{L_{D}^{2}+(Y p)^{2}}}
$$

$$
\theta=\tan ^{-1} \frac{Y p}{L_{D}}
$$

, where $(Y, Z)$ are the horizontal and vertical pixel coordinates of a detector image from the beam center, $p$ is the pixel size of the detector, and $L_{D}$ is the sample to detector distance. When the detector coordinate is given in the polar coordinate, 


$$
(Y, Z)=P(\cos \chi, \sin \chi)
$$

When we set $\frac{P p}{L_{p}} \equiv p_{0}$,

And

$$
q=2 k_{0} \sin \left(\frac{1}{2} \tan ^{-1} p_{0}\right)
$$

$$
\begin{gathered}
q_{x}=k_{0}\left(\frac{1}{\sqrt{p_{0}^{2}+1}}\left(\cos \phi-p_{0} \cos \chi \sin \phi\right)-\cos \phi\right) \\
q_{y}=k_{0}\left(\frac{1}{\sqrt{p_{0}^{2}+1}}\left(p_{0} \cos \chi \cos \phi+\sin \phi\right)-\sin \phi\right) \\
q_{z}=k_{0} \frac{p_{0} \sin \chi}{\sqrt{p_{0}^{2}+1}}
\end{gathered}
$$

Thus, for a given $\boldsymbol{q}$, the polar coordinate $\left(p_{0}, \chi, \phi\right)$ can be calculate as below:

$$
\begin{gathered}
p_{0}=\tan \left(2 \sin ^{-1} \frac{q}{2 k_{0}}\right) \\
\chi=\sin ^{-1}\left(\frac{q_{z}}{p_{0} k_{0}} \sqrt{1+p_{0}^{2}}\right) \\
\phi=\sin ^{-1}\left(\frac{\sqrt{p_{0}^{2}+1}}{k_{0}} \frac{\left(q_{y}\left(1-\sqrt{p_{0}^{2}+1}\right)-q_{x} p_{0} \cos \chi\right)}{\left(p_{0} \cos \chi\right)^{2}+\left(1-\sqrt{p_{0}^{2}+1}\right)^{2}}\right)
\end{gathered}
$$

For $\phi<0, \chi$ can be replaced with $\pi-\chi$ to get a positive $\phi$ value.

Using the relation, intensity at a pixel can be mapped into a position in the reciprocal space, or vise versa. Intensities were corrected for the polarization, solid angle, and background. Absorption correction was not applied because it was not practical to define the sample shape at various rotation angle.

c. Procedure for WAXS peaks in $\chi$ vs $\phi$ plot

A PbS NP has a fcc structure with a lattice constant of $\mathrm{a}_{\mathrm{PbS}}=5.96 \AA$. Its first two reflections, $\{111\}$ and $\{200\}$, are at $q_{111}=2 \pi / d_{111}$ and $q_{200}=2 \pi / d_{200}$, where $d_{\mathrm{hkl}}=\frac{\mathrm{a}_{\mathrm{Pbs}}}{\sqrt{h^{2}+k^{2}+l^{2}}}$. We fit azimuthal intensities shown in Figure $S 9$ with multiple Gaussian functions to find its max positions and plotted in Figure $2 d-f$ and j-I. 

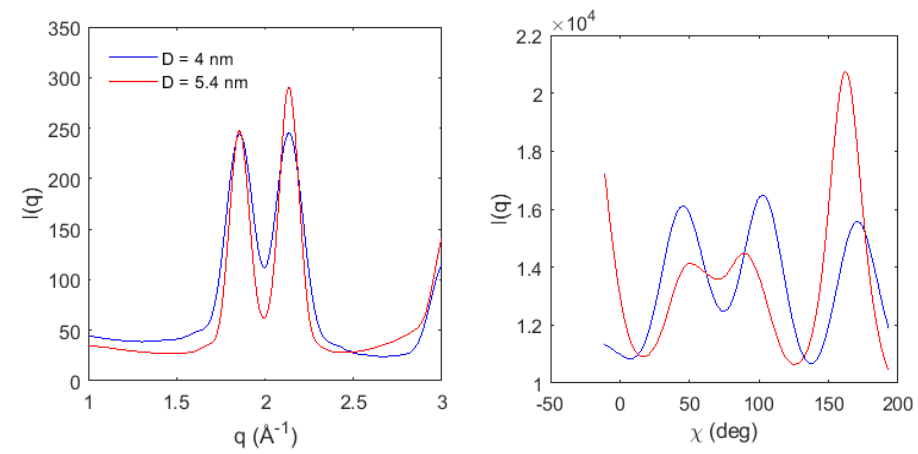

Figure S9. Intensity vs $q$ plot of WAXS of 4.14 and 5.6nm PbS NP (left) and azimuthal intensities of $\{111\}$ NP reflections (right). 


\section{Raw data}

a. SCs from 5.6nm PbS NPs:
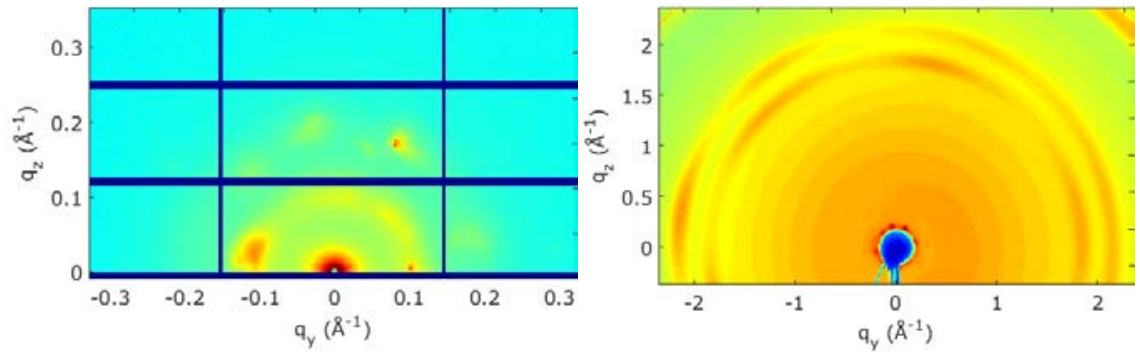

Figure S10. SAXS (left) and WAXS (right) data at $\phi=10$ degree.
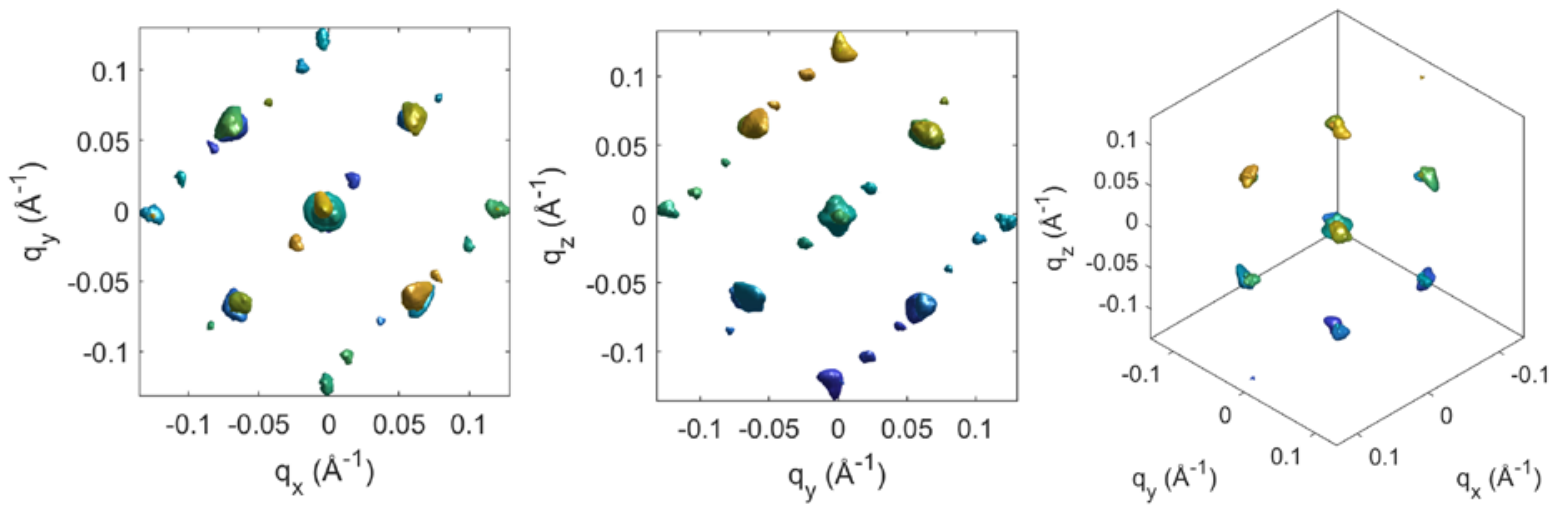

Figure S11. SAXS q map viewed along [001] SL, [100] SL, and [111] SL directions, from left to right, respectively.
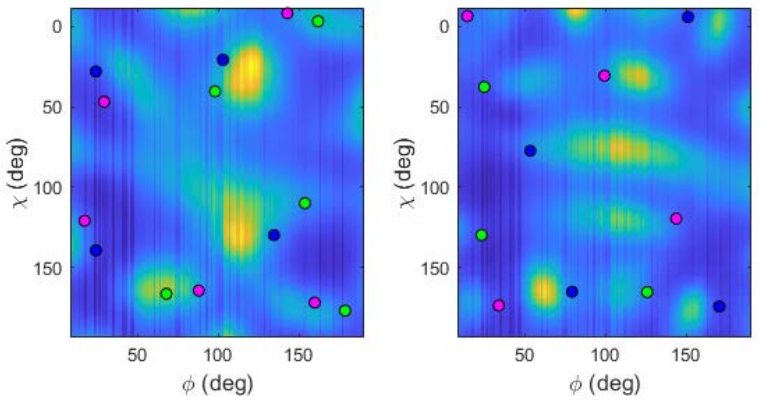

Figure S12. WAXS angle plots of $\{111\}$ NP (left) and $\{200\}$ NP (right) reflections. Color coded circles are positions of ideal reflections shown in Figure $2 \mathrm{~d}-\mathrm{f}$ for the models shown in Figure $2 \mathrm{c}$. 
b. SCs from $4.14 \mathrm{~nm}$ PbS NPs
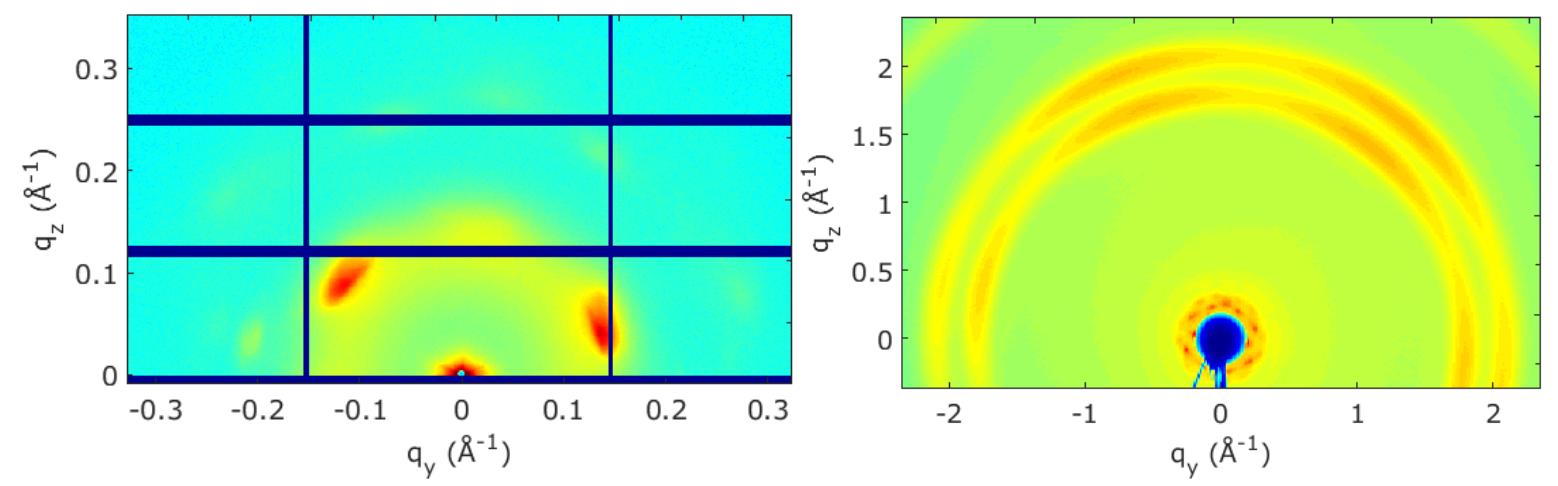

Figure S13. SAXS (left) and WAXS (right) data at $\phi=10$ degree.

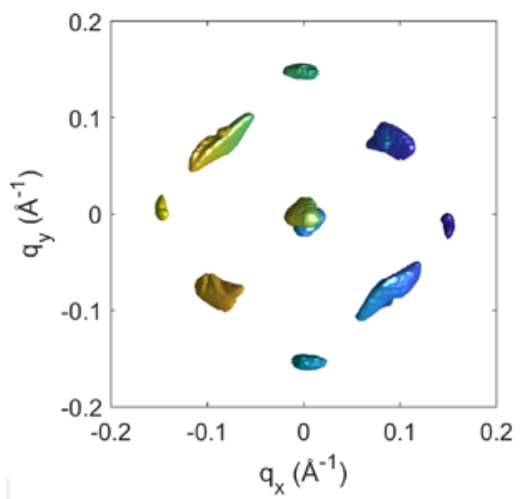

Figure S14. SAXS q map viewed along [001] SL.
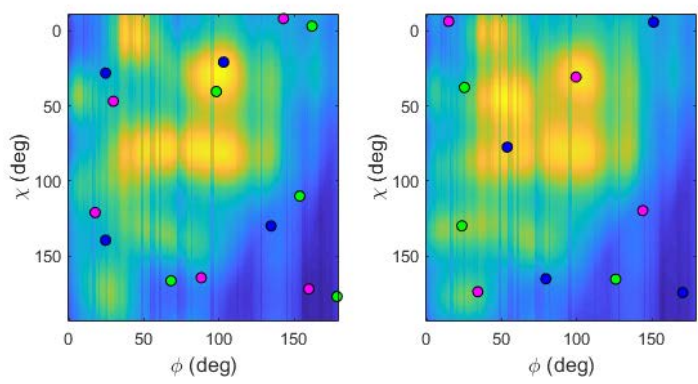

Figure S15. WAXS angle plots of $\{111\}$ NP (left) and $\{200\}$ NP (right) reflections. Color coded circles are positions of ideal reflections shown in Figures $2 \mathrm{j}-\mathrm{I}$ for the models shown in Figure $2 \mathrm{i}$. 


\section{Pole Figure for $4.14 \mathrm{~nm}$ PbS SC}

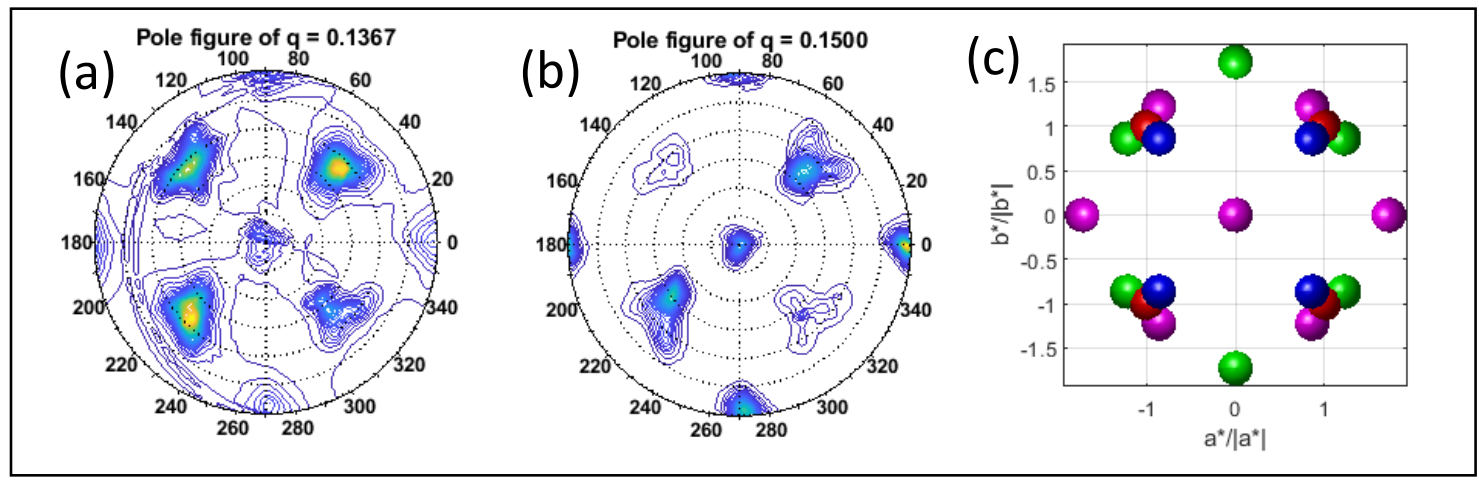

Figure S16. Pole figure plot of the reciproal space shown in Figure 2 at $q=0.1367 \AA^{-1}$ (a) and $0.15 \AA^{-1}$ (b). (c) is $\{111\}$ reflections of FCC (red), $\{110\}$ reflections of BCC structures of which $c^{*} / / q_{z}$ (blue), $c^{*} / / q_{x}$ (green), and BCC $\mathrm{c}^{*} / / q_{y}$ (magenta), where $\mathrm{c}^{*} / / q_{z}$ indicates that a reciprocal lattice vector $\mathrm{c}^{*}$ is parallel to $q_{z}$ axis. 


\section{0. $5.6 \mathrm{~nm}$ PbS NP SANS analysis}
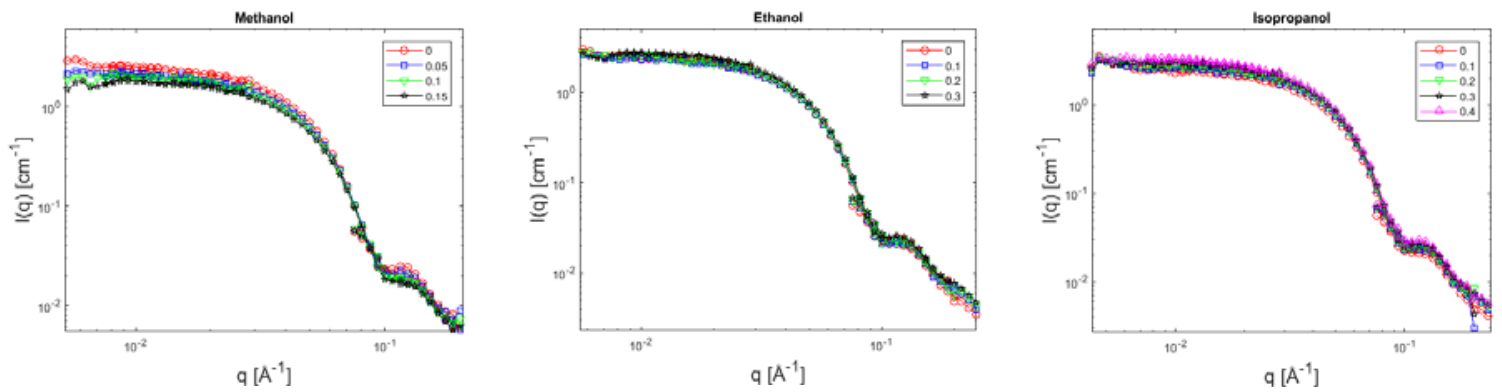

Figure S17. SANS raw data of 5.6nm PbS NP in toluene-d8 with methanol-d4, ethanol-d6 and isopropanol-d8, from left to right, respectively.

Table S3. Summary of the model fitting of SANS data

\begin{tabular}{|c|c|c|c|c|c|c|c|c|}
\hline & \multirow{2}{*}{$\begin{array}{c}\text { Alcohol } \\
\text { volume } \\
\text { fractio } \\
n(\%)\end{array}$} & \multirow{2}{*}{$\begin{array}{l}\text { Particle } \\
\text { Number } \\
\text { conc. }(f) \\
\left(\times 10^{15}\right. \\
\left.\mathrm{cm}^{-3}\right)\end{array}$} & \multirow{2}{*}{$\begin{array}{c}\text { Core } \\
\text { radius }^{\mathrm{a}} \\
\left(R_{C}\right)(\AA)\end{array}$} & \multirow{2}{*}{$\begin{array}{c}\text { Fractional } \\
\text { core-radius } \\
\text { distribution } \\
\text { width }^{\mathrm{a}} \\
\left(\sigma / R_{c}\right)\end{array}$} & \multirow{2}{*}{$\begin{array}{l}\text { Shell } \\
\text { thickness } \\
\left(t_{s}\right)(\AA)\end{array}$} & \multicolumn{3}{|c|}{ Scattering length density $\left(\times 10^{-6} \AA^{-2}\right)$} \\
\hline & & & & & & Core $\left(\rho_{c}\right)$ & Shell $\left(\rho_{s}\right)$ & $\begin{array}{l}\text { Solvent }^{\mathrm{b}} \\
\left(\rho_{\text {solv }}\right)\end{array}$ \\
\hline Alcohols & 0 & 9.15 & 27.16 & 0.145 & 13.6 & 2.343 & 0.584 & 5.664 \\
\hline \multirow{3}{*}{$\begin{array}{l}\text { Methanol- } \\
\text { d4 }\end{array}$} & 5 & 9.15 & 27.16 & 0.145 & 12.92 & 2.343 & 0.685 & 5.67 \\
\hline & 10 & 9.15 & 27.16 & 0.145 & 12.73 & 2.343 & 0.863 & 5.678 \\
\hline & 15 & 9.15 & 27.16 & 0.145 & 12.76 & 2.343 & 1.156 & 5.685 \\
\hline \multirow{3}{*}{ Ethanol-d6 } & 10 & 9.15 & 27.16 & 0.145 & 13.39 & 2.343 & 0.613 & 5.707 \\
\hline & 20 & 9.15 & 27.16 & 0.145 & 13.66 & 2.343 & 0.684 & 5.751 \\
\hline & 30 & 9.15 & 27.16 & 0.145 & 13.8 & 2.343 & 0.484 & 5.794 \\
\hline \multirow{4}{*}{$\begin{array}{c}\text { Isopropanol } \\
\text {-d8 }\end{array}$} & 10 & 9.15 & 27.16 & 0.145 & 13.28 & 2.343 & 0.122 & 5.647 \\
\hline & 20 & 9.15 & 27.16 & 0.145 & 13.51 & 2.343 & 0.07 & 5.631 \\
\hline & 30 & 9.15 & 27.16 & 0.145 & 14.01 & 2.343 & 0.07 & 5.614 \\
\hline & 40 & 9.15 & 27.16 & 0.145 & 14.6 & 2.343 & 0.07 & 5.597 \\
\hline
\end{tabular}

a. Obtained from SAXS

b. $\rho_{\text {solv }}=\phi_{t} \rho_{t}+\phi_{a} \rho_{a}$, where $\phi_{x}$ is the volume fraction of $x ; x=t$ and $a$ for toluene and alcohol, respectively. $\rho_{t}=5.664 \times 10^{-6} \AA^{-2}, \rho_{a}$ for methanol-d4, ethanol-d6 and isopropanol-d8 are 5.805 $\times 10^{-6} \AA^{-2}, 6.098 \times 10^{-6} \AA^{-2}$, and $5.497 \times 10^{-6} \AA^{-2}$, respectively.

In this model, we assume that the thickness of the shell is uniform as $t_{s}$. The size distribution of the core is the lognormal distribution function, which is

$$
n(x)=\frac{1}{\sqrt{2 \pi} R_{C} \sigma e^{\frac{1}{2} \sigma^{2}}} e^{-\frac{\log \left(\frac{x}{R_{C}}\right)^{2}}{2 \sigma^{2}}}
$$


SAXS and SANS scattering amplitude for a PbS NP is

$$
F=\left(\rho_{c}-\rho_{s}\right) F_{s}\left(q, x_{i}\right)+\left(\rho_{s}-\rho_{\text {solv }}\right) F_{s}\left(q, \beta x_{i}\right)
$$

, where $\beta=\left(R_{c}+t_{s}\right) / R_{c}$ and

, where $V(R)=\frac{4 \pi R^{3}}{3}$.

$$
F_{S}(q, R)=V(R) \frac{3(\sin (q R)-q R \cos (q R))}{(q R)^{3}}
$$

SAXS and SANS scattering intensity for a solution of PbS particles with a size distribution $n(x)$ is

$$
I(q)=f \int_{0}^{\infty} n(x)|F(q, x)|^{2} d x
$$

, where $f$ is the particle number concentration.

From $\rho_{s}$, volume fraction of toluene in the shell, $\phi_{t s}$ can be obtained; $\phi_{t s}=\left(\rho_{s}-\rho_{o}\right) /\left(\rho_{t}-\rho_{o}\right)$. $\rho_{o}$ is the scattering length density of oleic acids and is $0.078 \times 10^{-6} \AA^{-2}$, calculated with the density of 0.895 $\mathrm{g} / \mathrm{cm}^{3}$ using NIST scattering length density calculator. 


\section{Comments on solvent/non-solvent purification technique:}

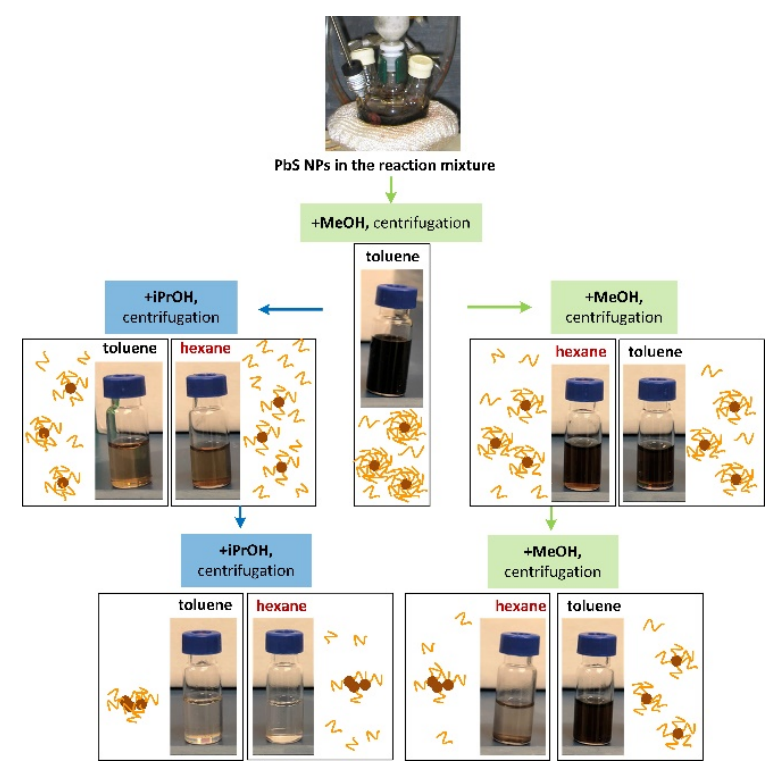

Figure S18. Optical images and depiction of the solvent/non-solvnet purification approach. For example, in the case of PbS NPs stabilized with oleic acids, methanol precipitates NPs from toluene more efficiently as compared to isopropanol. After PbS NPs were centrifuged after addition of isopropanol, there was a significant loss of NPs while almost full recovery of NPs was achieved by their precipitated with methanol as it is evidenced by more intense color of the toluene and hexane solutions. When NPs were dissolved in hexane, color of the recovered solution was not as intense as NPs that had been dissolved in toluene (compare the samples dissolved in hexane and toluene after the third purification step with methanol), suggesting significant recovery loss. We noticed aggregates at the bottom of the container in hexane, which could not be dispersed again. This indicates that the recovery loss is not because methanol is not an efficient non-solvent but because hexane that has higher miscibility with oleic acids than toluene can strip ligands off in the presence of non-solvents while, in toluene, ligands prefer to remain at the NP surface. As a result, use of hexane as a solvent in subsequent purification steps can lead the aggregation of NPs due to core-core interaction. 


\section{Reference}

1. Li, T.; Senesi, A. J.; Lee, B., Small Angle X-ray Scattering for Nanoparticle Research. Chem. Rev. 2016, 116 (18), 11128-11180.

2. Kriechbaum, M. Rg-Calculator of Polyhedra. https://www.staff.tugraz.at/manfred.kriechbaum/xitami/java/rgpolyhedra.html. 